\section{Orchidectomy alone for stage I testicular teratoma}

It has been common practice in the United Kingdom to recommend abdominal lymph node irradiation after orchidectomy for malignant teratoma of the testis, even in the absence of demonstrable abdominal disease. We present the details of 21 men whose only treatment at the time of presentation was orchidectomy. Their subsequent survival and pattern of relapse were compared to a similar group of irradiated patients to determine the benefits of prophylactic irradiation.

\section{Patients, methods, and results}

All patients were referred after radical orchidectomy. Clinical examination and chest radiography were the only staging procedures consistently adopted and no evidence of metastases was found. Twenty-one men seen between 1946 and 1973 received no further treatment at the time of referral. Histological specimens were reviewed at this hospital. Eighteen were malignant teratoma intermediate, two were malignant teratoma undifferentiated, and one was malignant teratoma trophoblastic. Between 1961 and 1970100 men received postoperative lymph node irradiation. Thirty-three had malignant teratoma intermediate, 39 malignant teratoma undifferentiated, two malignant teratoma trophoblastic, two mixed teratoma and trophoblastic, and 24 mixed teratoma and seminoma.

In more than two-thirds of the untreated patients radiotherapy was deferred because of recurring doubts about its efficacy. One patient was receiving peritoneal dialysis for unrelated chronic renal failure and another was severely mentally retarded. Two refused further treatment, and one was not referred until six months after orchidectomy. Fourteen patients remained alive and well. All deaths (7 of 21 ) occurred within two years of presentation and were due to metastatic disease. One patient died with uncontrolled para-aortic disease, six with widespread metastatic disease, and five with definite lung metastases.

Analysis of deceased patients from the untreated group

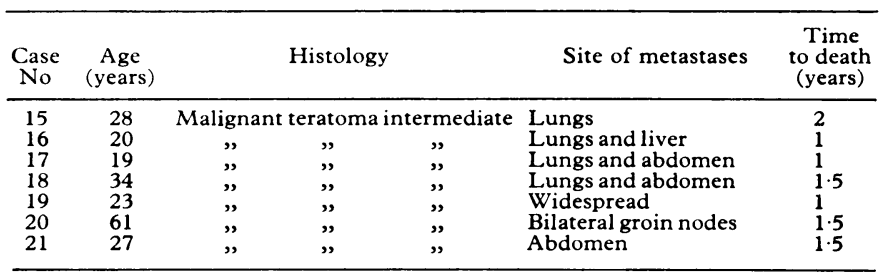

The irradiated patients received megavoltage radiotherapy on a $4 \mathrm{MeV}$ linear accelerator to the para-aortic and ipsilateral iliac nodes, the scrotum, and remaining testis. A mid-plane dose of $3500 \mathrm{cGy}$ in 20 daily fractions was given. Sixty-seven patients remained alive and well at five years. The five-year survival for malignant teratoma intermediate was 22 of 33 , and for malignant teratoma undifferentiated 28 of 39 . Most of those who died had pulmonary metastases and only four had appreciable para-aortic disease during their terminal illness.

\section{Comment}

The long-term survival of the untreated and irradiated patients was the same, $67 \%$. Although the untreated group was composed largely of malignant teratoma intermediate, this histology has not been associated with a better prognosis in those irradiated at this institute, where the five-year survival is $67 \%$ for malignant teratoma intermediate and $72 \%$ for malignant teratoma undifferentiated. Of those who died in the untreated group, half had appreciable paraaortic disease compared with only $12 \%$ of the irradiated patients who died. Nevertheless, the same proportion of patients from each group died of widespread disease and most had blood-borne metastases, particularly affecting the lungs.

Prophylactic abdominal irradiation had no apparent influence on the ultimate fate of our patients. Two-thirds must have been cured by orchidectomy alone, a figure supported by evidence from American series of retroperitoneal block dissections. ${ }^{1}$ It has been suggested that adjuvant chemotherapy merits exploration in those whose disease is apparently confined to the testis (stage I). ${ }^{2}$ 'This would subject more than two-thirds of our stage I patients to unnecessary, intensive chemotherapy with its attendant morbidity and unknown long-term risks. A policy of careful staging ${ }^{34}$ and close follow-up with recourse to effective chemotherapy ${ }^{5}$ at the first sign of relapse has much to commend it. Chemotherapy now offers a real possibility of cure for these patients who relapse and unnecessary irradiation and exposure to cytotoxic drugs could be avoided in most cases.

${ }^{1}$ Maier JG, Buskirk KE, Sulak MH, Perry RH, Schamber DT. An evaluation of lymphadenectomy in the treatment of malignant testicular germ cell neoplasms. F Urol 1969;101:356-9.

2 Slater AJ, James KW, Fifield R, Green GV. The investigation and treatment of germ cell tumours of the testis. Clin Radiol 1981 ;32: 25-30.

${ }^{3}$ Scardino PT, Cox HD, Waldman TA, McIntyre KR, Mittemeyer B, Javadpour $N$. The value of serum tumour markers in the staging and prognosis of germ cell tumours of the testis. 7 Urol 1977;118:994-9.

4 Husband JE, Peckham MJ, MacDonald JS, Hendry WF. The role of computed tomography in the management of testicular teratoma. Clin Radiol $1979 ; 30: 243-52$.

${ }^{5}$ Einhorn LH, Donohue JP. Improved chemotherapy in disseminated testicular cancer. $\mathcal{F}$ Urol $1977 ; 117: 65-9$.

(Accepted 23 fune 1981)

Christie Hospital and Holt Radium Institute, Manchester M20 9BX R STOUT, MRCP, FRCR, senior registrar in radiotherapy and oncology R D HUNTER, MRCP, FRCR, consultant in radiotherapy and oncology

\section{Clonazepam: effective treatment for restless legs syndrome in uraemia}

Uraemia is the most common cause of the restless legs syndrome and in our experience occurs in $15-20 \%$ of patients receiving dialysis. For many patients it is their most unpleasant and distressing symptom, preventing sleep and rarely responding to treatment. In the idiopathic syndrome, Matthews ${ }^{1}$ found that clonazepam relieved the symptoms. We have therefore evaluated the drug in the uraemic restless legs syndrome.

\section{Patients, methods, and results}

We studied 15 patients aged 18-66 years ( 11 men, four women), of whom 10 were receiving maintenance haemodialysis for terminal renal failure and five were being treated for chronic renal failure. Durations of dialysis ranged from less than one to seven years. Severe restless legs had been present continuously in 13 patients for six months to five years and was intermittent in the other two. All were taking iron and folate supplements regularly and none had overt symptoms or signs of peripheral neuropathy. In all cases the symptoms were worse in the evening and at night. Most had been treated ineffectively with various sedatives and hypnotics, including temazepam, chlordiazepoxide, diazepam, nitrazepam, and lorazepam.

Clonazepam was usually given as a split evening dose of $0.5 \mathrm{mg}$ at $6 \mathrm{pm}$ and a further $0.5 \mathrm{mg}$ half an hour before retiring, or as a more conventional twice-daily dosage for those with diurnal symptoms. The dosage was increased as necessary. Four patients were initially treated for one month with a similar regimen using diazepam $5 \mathrm{mg}$ before the clonazepam was started, and in two patients the diazepam was substituted for the clonazepam after a month's successful treatment. Dialysis regimens remained unaltered throughout.

In all but one patient response was swift and complete. There was total abolition of the symptoms after the first dose of clonazepam in six of the patients; eight others responded to an increase in the dosage. Only one patient required a daily dose of clonazepam of more than $2 \mathrm{mg}$, and his response was incomplete. Diazepam given either before or after clonazepam failed to suppress the symptoms. The two patients whose symptoms had been suppressed with clonazepam which was then switched to diazepam found a return of their symptoms so distressing that they changed back to clonazepam after two and four days respectively. Other than somnolence, which was pronounced in only one patient, no adverse side effects were encountered. This patient's symptoms were only partially suppressed by clonazepam, $3 \mathrm{mg}$ daily, higher doses rendering him too lethargic to drive his car the next morning. 